\title{
Shear Modulus Titration in Crystalline Colloidal Suspensions
}

\author{
Thomas Palberg, ${ }^{1}$ Johannes Kottal, Franz Bitzer, Rolf Simon, Mathias Wüth, and Paul Leiderer \\ Universität Konstanz, Fakultät fur Physik, D-78434 Konstance, Germany
}

Received December 14, 1993; accepted May 31, 1994

\begin{abstract}
We present the first direct experimental access to the actual surface charge number $Z$ of colloidal particles under conditions of strong electrostatic interaction. We further calculate a renormalized charge number $Z^{*}(Z)$ using the modified DLVO approximation and the dependence of the shear modulus $G\left(Z^{*}\right)$ on the concentration of neutral electrolyte $n_{s}$. The excellent agreement of predicted and measured values provides an experimental verification of the renormalization concept under variation of the salt concentration. The link between the true surface charge number and experimental results via $Z^{*}$ is extended to the conductivity $\sigma\left(Z^{*}\right)$. On the basis of the number of dissociable surface groups $N$ and numerical solutions of the nonlinearized Poisson-Boltzmann equation we further provide a qualitative model for incomplete dissociation under conditions of overlapping double layers.
\end{abstract}

\section{INTRODUCTION}

Suspensions of latex spheres in aqueous electrolytes may be synthesized with well-defined mean diameters and surface properties. Systems with very small variations in particle properties, so-called monodisperse colloids, have been widely used as model suspensions within the extremely heterogeneous class of colloidal dispersions which is of prime technical and biological importance (1-3).

Under certain experimental conditions of high interparticle interaction such suspensions show fluid or crystalline ordered phases. For charged systems these are observed for high surface charge number $Z \sim 10^{3}$, a low concentration of electrolyte $c_{\mathrm{s}} \leqslant 10^{-5} \mathrm{~mol} \mathrm{liter}^{-1}$, and a sufficiently high volume fraction $\Phi \geqslant 0.005(4,5)$. Since typical particle spacings are on the order of the wavelength of visible light, most structural and dynamical properties of these systems are accessible by optical techniques. Although similar to atomically condensed matter this so-called soft matter exhibits a number of specific and interesting differences (1-3, 6-8).

Due to the low volume fraction, the elastic moduli of crystalline suspensions are on the order of $0.1-1 \mathrm{~Pa} \mathrm{(6)}$. Colloidal crystals therefore are very fragile objects which may easily

\footnotetext{
${ }^{1}$ To whom correspondence should be addressed.
}

be transformed to a shear molten state simply by shaking the sample (7). Due to the presence of the viscous suspending medium the typical relevant time scales for crystallization (7), vibration (6), or long time self diffusion (8) are close to each other and to the time scale of free Brownian motion. Therefore a number of general problems have been addressed in these systems which are not as convenient to observe in other condensed matter, e.g., the real time study of solidification processes on a microscopic level. The observed properties are well described using Yukawa type potentials (1-3) for which a number of alternative formulations are available. An analytically simple description is given by the so-called modified Dejarguin-Landau-Verwey-Overbeek (DLVO) approximation (MDA) (9). It uses only the repulsive part of the DLVO potential. The energy of interaction between a pair of spherical particles of radius $a$ and true surface charge number $Z$ at a separation $d$ and immersed in a medium of dielectric permitivity $\epsilon=\epsilon_{0} \epsilon_{\mathrm{H}_{2} \mathrm{O}}$ is given by

$$
V(d)=\frac{Z^{2} e^{2}}{4 \pi \epsilon}\left(\frac{\exp (\kappa a)}{(1+\kappa a)}\right)^{2} \frac{\exp (-\kappa d)}{d} \text {. }
$$

The Debye screening parameter $\kappa$ is strongly dependent on the bulk number density $n_{i}$ and charge $z_{i}$ of the small ions including the counterions,

$$
\kappa=\left(\frac{e^{2}}{\epsilon k_{\mathrm{B}} T} \sum n_{i} z_{i}^{2}\right)^{1 / 2}
$$

where $T, k_{\mathrm{B}}$, and $e$ are the temperature, the Boltzmann constant, and the elementary charge, respectively. The bulk number density is given for the protons by $n_{\mathrm{H}^{+}}=n_{\mathrm{p}} Z$ and for each excess salt ion by $n_{i}=1000 c_{i} / N_{\mathrm{A}}$, where $c_{i}$ is the molar concentration and $N_{\mathrm{A}}$ the Avogadro constant.

This solution of the linearized Poisson-Boltzmann (PB) equation is strictly valid only in the case of small surface potentials, corresponding to very low true surface charge numbers. For higher charge numbers the MDA replaces $Z$ by a somewhat smaller renormalized charge $Z^{*}(9,10)$ and the proton number density is given by $n_{\mathrm{H}^{+}}=n_{\mathrm{p}} Z^{*}$. The renormalized charge $Z^{*}$ is calculated numerically and varies in specific ways in dependence on true charge number $Z$, 
salt concentration $c_{\mathrm{s}}$, and volume fraction $\Phi(11)$. This description will elsewhere be shown to hold in ordered colloids under conditions of constant true surface charge and strong interparticle interaction, where the particles do not get close to contact (10). MDA and other Yukawa potentials have been used with the charge as a free fit parameter to interprete the elastic properties of colloidal crystals (6) or the structure of fluid systems $(12,13)$. While all other parameters $(a, \Phi$, $n_{\mathrm{s}}$ ) were extracted from independent measurements and $k$ was calculated from bulk values, at least an effective charge could be determined. However, the prediction of suspension properties from measured true surface charges has, as yet, rarely been performed.

First, because there was no experimental access to the true surface charge $Z$ under conditions of strong interparticle interaction so far. While conductometric titration only gives the total number of surface groups $N$, irrespective of their degree of dissociation (14), the interpretation of electrophoretic measurements currently lacks an electrokinetic theory for electrostatically interacting particles (13). The comparison of static structure factors after addition of $\mathrm{NaOH}$ to those measured after addition of neutral $\mathrm{NaCl}$ has been tried, but under the experimental conditions involved $\left(c_{\mathrm{NaOH}}>\right.$ $c_{\mathrm{eq}}$ ) only the total number of dissociable groups $N$ was accessible (15).

Second, for highly charged particles $Z$ may differ considerably from the single-particle case due to the overlap of the particle double layers. Using particles with variable number of surface groups it has recently been shown that a self-consistent description of phase behavior and transport properties in the ordered state can be achieved only if details of the surface chemistry are known (16). Bucci et al. successfully described the static structure factors of a micellar system equating the true surface charge to the low number of surface groups $(N \approx 50)$ known from the micellar stoechiometry (17). However, such additional information usually is missing and there is need for a charge characterization technique which gives in situ access to $Z, Z^{*}$, and $N$.

In order to provide this experimental link between particle and suspension properties we present here the shear modulus titration of colloidal crystals. We first determine the number of true surface charges $Z$, then derive a renormalized charge number $Z^{*}(Z)$, and finally compare calculated shear moduli $G\left(Z^{*}\right)$ and conductivities $\sigma\left(Z^{*}\right)$ to experimental values measured in dependence on the concentration of neutral electrolyte, respectively, $\mathrm{NaOH}$.

\section{EXPERIMENTAL}

The concept of titrating an interaction-dependent property constitutes an experimental challenge that is solved only on the basis of precise control of experimental conditions and a high accuracy of the measurements themselves.

We used commercially available particles of hydrodynamic radius $a_{\mathrm{h}}=51 \mathrm{~nm}$ (LOT 2011M9R, Seradyn, Inc.,
USA) carrying sulfate and carboxylate surface groups. The experimental conditions were first adjusted to zero concentration of excess electrolyte using a recently reported advanced deionization technique (18). $\mathrm{NaOH}$ (p.a. grade) was then added in small quantities and the resulting micromolar concentrations controlled via conductivity. The particle density $n_{\mathrm{p}}$ was adjusted to $n_{\mathrm{p}}=5.4 \times 10^{18} \mathrm{~m}^{-3}$ (volume fraction $\Phi=0.0030$ ) and was controlled via measurements of the static structure factor. Errors in the experimental conditions are below $1 \%(18)$.

After deionization the suspension is left in a shear molten state from which it readily crystallizes to form an untextured polycrystalline solid. Other morphologies can be prepared by shear processing during or after complete solidification (19). For the untextured polycrystalline materials the shear moduli dependent on the concentration of added electrolyte were measured by time-resolved static light scattering. The method has been reported in detail elsewhere (19). We note here that we detect the resonance spectra of the colloidal solids excited to torsional vibrations by recording the periodic shifts of the Bragg condition under oscillatory shear. Knowing the sample geometry, the shear modulus $G$ is derived from the resonance frequencies which in turn are connected to the pair energy of interaction by $(6,19)$

$$
G=f_{\mathrm{a}} f_{\mathrm{s}} n_{\mathrm{p}} \kappa^{2} d^{2} V(d) .
$$

Here $f_{\mathrm{s}}$ and $f_{\mathrm{a}}$ are known numerical factors depending on the crystal structure and the average of their relative orientation as compared to the direction of applied shear. The method is accurate enough to monitor even slight changes in sample morphology and the reproducibility of $G$ is better than $2 \%$ in the range of $G \sim 0.1 \mathrm{~Pa}(19)$.

Upon the addition of neutral electrolyte the shear modulus decreases slowly until at the phase transition to the fluid state it drops to zero. Simultaneously the conductivity $\sigma$ rises linearily with the salt concentration $c_{\mathrm{s}}$ and may be described by assuming independent ion migration for the strongly interacting system $[13,17]$

$$
\sigma=Z_{\mathrm{tr}} e n_{\mathrm{p}}\left(\mu_{\mathrm{p}}+\mu_{\mathrm{H}^{+}}\right)+\sum n_{i} e \mu_{\mathrm{j}} .
$$

$Z_{\mathrm{tr}}$ is an effectively transported charge that can be derived, if the small ion mobilities $\mu_{\mathrm{i}}$ are taken from literature (bulk values!) and the particle mobility $\mu_{\mathrm{p}}$ is known from, e.g., laser Doppler anemometry. Although there is strong experimental evidence for the validity of such a description in the case of significantly overlapping double layers, we note that this point still calls for detailed theoretical clarification. We further comment on this under Results.

Titrations with freshly prepared $\mathrm{NaOH}$ are carried out under an inert gas atmosphere while the tubes and cells containing the suspension were carefully sealed against leakage of $\mathrm{CO}_{2}$. Upon addition of $\mathrm{NaOH}$ to a completely deionized 


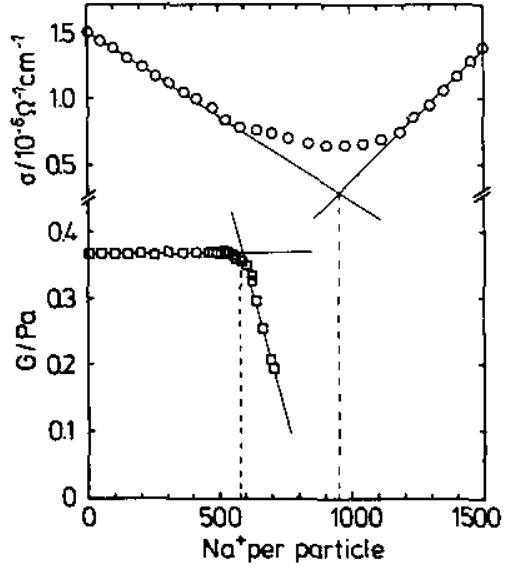

FIG. 1. Titrations performed simultaneously on a crystalline suspension of polystyrene latex spheres of diameter $102 \mathrm{~nm}$ at a particle density of 5.4 $\times 10^{18} \mathrm{~m}^{-3}$. The conductivity $\sigma$ (upper scale, $\mathrm{O}$ ) and the shear modulus $G$ (lower scale, $\square$ ) are dependent on the concentration of $\mathrm{Na}^{+}$ions per particle. Solid lines are fits to extrapolate the equivalent points. From the conductivity titration we find a total number of dissociable surface groups $N=950$. The shear modulus titration yields a true surface charge number of $Z=580$.

suspension of particles carrying incompletely dissociated acid groups in a first step, an exchange of the highly mobile dissociated $\mathrm{H}^{+}$counterions by $\mathrm{Na}^{+}$ions occurs. During this process the degree of dissociation is not changed. The weaker the acid the earlier an excess concentration of $\mathrm{NaOH}$ is built up which causes further dissociation of the acid groups (14).

\section{RESULTS}

Both shear modulus measurements under deionized conditions and conductometric titrations at elevated salt concentrations have extensively been used before to characterize the charge of colloidal particles $(6,14)$. We here present a novel approach and monitor both elastic and transport properties during the titration process. The developments of the shear modulus and of the conductivity as a function of the amount of added $\mathrm{Na}^{+}$per particle are shown in Fig. 1 .

We observe the expected initially linear drop in the conductivity (14). The decrease in $\sigma$ then continues sublinearily and an inflection point is reached. Finally, after complete neutralization, the conductivity increases linearily due to the addition of $\mathrm{NaOH}$. As usual the equivalence point is inferred from extrapolation of the two linear regimes. The number of dissociable sulfate groups is calculated as $N=$ $n_{\mathrm{Na}^{+}}+$(eq.) $/ n_{\mathrm{p}}=950$. A second inflection point at $N_{\mathrm{COOH}}=$ $2 \times 10^{4}$ is not shown, since the carboxylate groups remain undissociated under the conditions examined here (surface pH < 4) (14). The experimental error in $N$ of less than $5 \%$ is practically due to the extrapolation procedure only.

The shear modulus $G$ remains constant until $580 \mathrm{Na}^{+}$per particle are added. This implies that also the surface charge $Z$ and the total number of screening ions remains constant.
Neutralization starts with the unbound counterions, i.e., the dissociated protons constituting the electrostatic double layer. Within the experimental error we do not detect a difference in the interaction between particles surrounded by a double layer of $\mathrm{H}^{+}$and of $\mathrm{Na}^{+}$. This provides a strong evidence for the validity of a Yukawa-type description which actually neglects the differences in ionic radii of the small ions.

Since we know that $\mathrm{Na}^{+}$does not show specific adsorption on polystyrene (14) and the active surface groups are sulfate groups, we may safely assume their dissociation to be the dominant charge-determining mechanism and neglect more complicated surface processes. We therefore equate the amount of $\mathrm{Na}^{+}$added at the start of the decrease in $G$ with the true number of surface charges $Z=580$. (For the case of specific adsorption or complexation of surface groups we would detect the number of free protons outside the outer Helmholtz plane (14).)

The observed decrease in the shear modulus at higher electrolyte concentration is somewhat slower than that in the case of neutral salt. This indicates the only partial use of $\mathrm{NaOH}$ to form the increasing electrolyte background, while the rest is used up in the simultaneously continuing neutralization reaction. The suspension finally melts upon the addition of $720 \mathrm{Na}^{+}$per particle.

In order to predict the elastic properties of colloidal crystals we first numerically determine the renormalized charge $Z^{*}$ from the true surface charge number $Z=580$ (11, using a program kindly provided by $\mathrm{W}$. Härtl). We find $Z^{*}(Z)=$ 390 for the completely deionized state respectively for the addition of less than $580 \mathrm{Na}^{+}$per particle. The procedure is repeated for finite concentrations of excess neutral electrolyte. Inserting $Z^{*}$ into Eqs. [1] and [2] we then calculated the shear modulus for an unoriented polycrystalline colloidal solid dependent on the electrolyte concentration using Eq. [3].

Figure 2 shows a comparison of the MDA prediction to the experimental data. Excellent agreement is observed between the results of the shear modulus measurements and

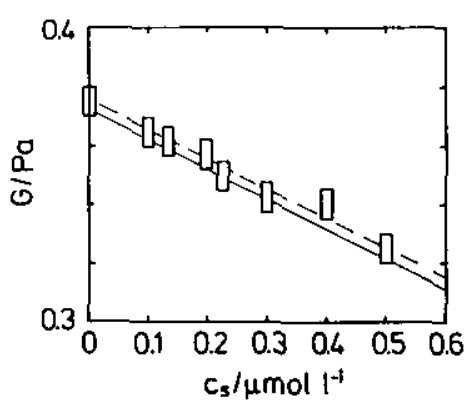

FIG. 2. The dependence of the shear modulus $G$ of the same suspension described in the legend to Fig. 1 on the concentration of neutral electrolyte ( $\mathrm{NaCl}$ ). $\square$, experimental data; - , values calculated via MDA using $Z=$ $580, Z^{*}=390$, and $n_{\mathrm{s}}=Z^{*} n_{\mathrm{p}}+n_{\mathrm{Na}^{+}}+n_{\mathrm{Cl}^{-}}$, respectively; -- , best fit of a modified DLVO to the data yielding an effective charge of $Z_{\mathrm{efF}}=395$. 


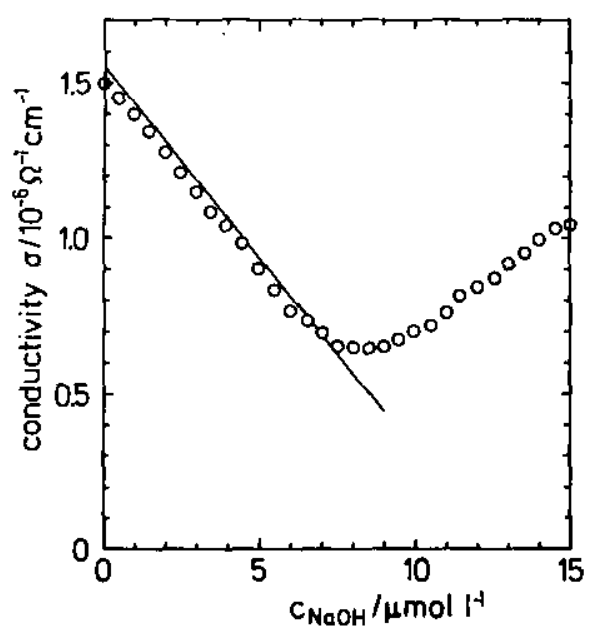

FIG. 3. The dependence of the conductivity $\sigma\left(Z^{*}\right)$ of the same suspension described in the legend to Fig. 1 on the concentration of $\mathrm{NaOH} . \mathrm{O}$, experimental data; - , calculated values using $Z_{\mathrm{tr}}=Z^{*}=390$ in Eq. [4].

the prediction. Deviations are within the experimental error of $2 \%$. Note that the prediction does not contain any free parameter. The best fit of Eq. [3] using an MDA potential to the experimental data yields an effective charge of $Z_{\text {eff }}=$ 395 and is also included in Fig. 2. We state a quantitative agreement between the calculated renormalized and the fitted effective charge number: $Z^{*}=Z_{\text {eff }}$. Thus the elastic properties of a highly charged colloidal system may be precisely predicted via the renormalization procedure performed on the basis of accurately determined $Z, n_{\mathrm{p}}$, and $n_{\mathrm{s}}$.

This is the first unambiguous link between independently determined true surface charges on one side and an interaction-dependent property of an ordered colloidal suspension on the other. The charge number of strongly interacting colloidal spheres is no free fit parameter any more.

We also tried predictions and fits with other potentials. An equally good performance was observed for the renormalized Yukawa potential, which neglects the finite size of the particle and calculates the screening parameter from the small ion density at the Wigner-Seitz cell boundary (11). A DLVO potential used without charge renormalization overestimates the rigidity of the colloidal solid by some $40 \%$.

We further comment on the application of Eq. [4], describing the conductivity of suspensions under conditions of overlapping double layers. The electrophoretic mobility of the particles at no added salt and $n_{\mathrm{p}}=4 \times 10^{18} \mathrm{~m}^{-3}$ is $\mu_{\mathrm{p}}$ $\approx 10 \times 10^{-8} \mathrm{~m}^{2} \mathrm{~V}^{-1} \mathrm{~s}^{-1}(20)$. Using $Z^{*}(Z)$ as input for Eq. [4] we calculated the conductivity of the suspension during the initial decrease observed upon titration with $\mathrm{NaOH}$. As is shown in Fig. 3 the calculated values are only slightly larger than the experimentally observed values. The deviations are less than $5 \%$ in the region of linear decrease. Thus the knowledge of the true surface charge enables a reasonable estimate via the charge renormalization procedure also for an important transport property of ordered colloidal suspensions.

Albeit this agreement between experimental data and Eq. [4] is not accidental and has been observed in a number of cases $(13,19)$, it is in fact not well understood, since a lot of unproven assumptions are involved in the use of Eq. [4]. In particular the use of the renormalized charge is as yet not justified on theoretical grounds and the development of a rigorous description of transport processes in systems with overlapping double layers would be extremely helpful. Nevertheless we may use Eq. [4] in both deriving effectively transported charges and predicting conductivity values.

Table 1 summarizes the results gathered from this single titration measurement. Effective charge numbers derived from the shear modulus excellently agree with the renormalized charges calculated from the true surface charge number via the renormalization procedure. Also the effectively transported charge numbers are very close to this value.

In addition to the characterization of charges we may obtain some further information about the surface chemistry of the particles from the conductivity titration. Only 580 of 950 sulfate groups are dissociated and the degree of dissociation is $\alpha=Z / N=0.61$. The dissociation equilibrium even in dilute colloidal systems is significantly altered as compared to the situation in simple electrolytes. The complications arise because of the inhomogeneous distribution of small ions and one has to impose the restrictions of charge balance and potential decay within the double layer. Instead of bulk values, the description in terms of the mass equation uses surface concentrations, i.e., $\left[\mathrm{H}^{+}\right]_{\mathrm{s}}=\left[\mathrm{H}^{+}\right] \exp \left(-e \Psi_{0} /\right.$ $k_{\mathrm{B}} T$ ), where $\Psi_{0}$ is the surface potential (14). From this we may calculate the dissociation constant of the sulfate surface groups to be $K_{\mathrm{SO}_{4} \mathrm{H}}=\left[\mathrm{SO}_{4}^{-}\right]\left[\mathrm{H}^{+}\right] /\left[\mathrm{SO}_{4} \mathrm{H}\right]=\alpha\left[\mathrm{H}^{+}\right]_{s} /$ $(1-\alpha)$.

Following the procedure valid for noninteracting particles (14), we take a surface potential $\Psi_{0}=Z e / 4 \pi \epsilon_{0} a(1+\kappa a)$ $=0.199 \mathrm{~V}$, and the bulk proton concentration $\left[\mathrm{H}^{+}\right]=5.2$

TABLE 1

Comparison of Different Charge Numbers for a Suspension of Crystalline-Ordered Polystyrene Latex Spheres of Radius $a$ $=51 \mathrm{~nm}$ at a Particle Density of $n_{\mathrm{P}}=5.4 \times 10^{18} \mathrm{~m}^{-3}$

\begin{tabular}{llccc}
\hline $\mathrm{N}_{\mathrm{SO}_{4}}$ & $Z$ & $Z^{*}(\mathrm{PBC})$ & $Z_{\mathrm{eff}(G)}$ & $Z_{\mathrm{tr}}(\sigma)$ \\
\hline 950 & 580 & 390 & 395 & 385 \\
\hline
\end{tabular}

Note. $\mathrm{N}_{\mathrm{SO}_{4}}$, number of dissociable sulfate groups determined by conductometric titration; $Z$, number of dissociated groups determined from shear modulus titration; $Z^{*}(\mathrm{PBC})$, renormalized charge number determined from fits of a Yukawa potential to the numerical solution of the Poisson-Boltzmann equation based on $Z(11) ; Z_{\text {eff }}(G)$, effective charge number determined from a fit to the experimentally determined shear modulus $G$ using the MDA; $Z_{\mathrm{tr}}(\sigma)$, effectively transported charge determined from a fit of Eq. [4] to the conductivity data. 
$10^{-6} \mathrm{~mol} \mathrm{liter}^{-1}$ to get a surface proton concentration of $\left[\mathrm{H}^{+}\right]_{\mathrm{s}}=1.5310^{-2} \mathrm{~mol} \mathrm{liter}^{-1}$ and from that $\mathrm{p} K_{\mathrm{s}}=1.62$ which is slightly lower than the surface $\mathrm{pH}_{\mathrm{s}}=1.81$. The numerical solution of the PB equation for interacting colloidal spheres yields a surface concentration of $\left[\mathrm{H}^{+}\right]_{\mathrm{s}}=1.42$ $10^{-2}$ mol liter $^{-1}$. From this we derive a $\mathrm{p} K_{\mathrm{s}}$ of 1.67 , which is of the same order as the value obtained before and in good agreement with literature values for similar latex spheres (14).

For an isolated particle with completely dissociated groups the surface $\mathrm{pH}$ is well above the $\mathrm{p} K_{\mathrm{s}}$. Note, however, that under conditions of overlapping double layers the surface concentration of protons is drastically increased as compared to that of the dilute system. Since the dissociation equilibrium is highly sensitive to small changes in $\left[\mathrm{H}^{+}\right]$once the surface $\mathrm{pH}$ gets close to the $\mathrm{p} K_{\mathrm{s}}$, we observe a reduced true surface charge number $Z$ as compared to that of the dilute, noninteracting case.

To capture this effect of particle correlation on the surface chemistry we suggest a formulation following the concept of renormalization. We calculate both the bulk and surface concentrations of protons using $Z^{*}$ and as before a DebyeHückel potential to obtain $\left[\mathrm{H}^{+}\right]^{*}=3.5410^{-6} \mathrm{~mol} \mathrm{liter}^{-1}$, $\Psi_{0}^{*}=0.136 \mathrm{~V},\left[\mathrm{H}^{+}\right]_{\mathrm{s}}^{*}=8.3110^{-4} \mathrm{~mol} \mathrm{liter}^{-1}$, and $\mathrm{pH}_{\mathrm{s}}^{*}=$ 3.08. Since we reduce the surface charge from $N=950$ to $Z^{*}=390$ we obtain a renormalized value for the $\mathrm{p} K$ of $\mathrm{p} K^{*}=3.32$. This formulation nicely reflects the observation that under conditions of strong particle interaction the apparent chemical behavior, even of $\mathrm{SO}_{4}$ surface groups, is that of weak acids.

\section{CONCLUSION}

As shown in the last section, the charge number of colloidal particles sensitively depends on the degree of overlapping double layers. A decrease of the degree of dissociation as compared to that of the noninteracting case may occur with increasing volume fraction or decreasing salt concentration. The titration of an interaction-dependent property gives access to the true surface charge number $Z$ of colloidal suspensions under conditions of strongly overlapping double layers. Using the novel method of shear modulus titration $Z$ may be determined with an accuracy better than $5 \%$. Supplementary conductivity titration yielded further information about the surface chemical properties of the particles.

A renormalized charge number $Z^{*}$ may be calculated from the true surface charge and used with the MDA to predict the properties of strongly interacting colloidal suspensions. Both the shear modulus and the conductivity were measured in dependence on the concentration of added electrolyte. We observed excellent agreement of the predictions with the experimentally determined elastic and transport properties. Vice versa both measurements easily yield quantitative estimates of the effective charge.

\section{ACKNOWLEDGMENTS}

We thank H. Löwen, M. Deggelmann, T. Ghisler, and P. Schurtenberger for many inspiring discussions. Financial support from the DFG is gratefully acknowledged.

\section{REFERENCES}

1. Pusey, P. N., in "Liquids, Freezing and Glass Transition: Ecole d'ete, Les Houches, 51st, 1989" (J. P. Hansen, D. Levesque, and J. ZinnJustin, Eds.), p. 763. Elsevier, Amsterdam, 1991.

2. Safran, S. A., and Clark, N. A. (Eds.), "Physics of Complex and Supramolecular Fluids." Wiley-Interscience, New York, 1987.

3. "Cristeaux Colloideaux," J. Phys. Colloq. C3-46 (1985).

4. Voegtli, L. P., and Zukoski, C. F., J. Colloid Interface Sci. 141, 79 (1990).

5. Meijer, E. J., and Frenkel, D., J. Chem. Phys. 94, 2269 (1991).

6. Lindsay, H. M., and Chaikin, P. M., in "Physics of Complex and Supramolecular Fluids" (S. A. Safran and N. A. Clark, Eds.), p. 269. WileyInterscience, New York, 1987.

7. Ackerson, B. J. (Ed.), Phase Transitions 21 (2-4) (1990).

8. Simon, R., Palberg, T., Leiderer, P., J. Chem. Phys. 99, 3030 (1993).

9. Dozier, W. D., Lindsay, H. M., and Chaikin, P. M., J. Phys. Colloq. C3-46, 257 (1985).

10. Bitzer, F., Palberg, T., Löwen, H., Simon, R., Leiderer, P., Phys. Rev. E 50(4), 2252 (1994).

11. Alexander, S., Chaikin, P. M., Grant, P., Morales, G. J., Pincus, P., and Hone, D., J. Chem. Phys. 80, 5776 (1984).

12. Härtl, W., and Versmold, H., J. Chem. Phys. 88, 7157 (1988).

13. Deggelmann, M., Palberg, T., Hagenbüchle, M., Maier, E. E., Krause, R., Graf, Ch., and Weber, R., J. Colloid Interface Sci. 143, 318 (1991); J. Phys. Condens. Matter 4, 3077 (1992).

14. James, R. O., Davis, J. A., and Leckie, J. O., J. Colloid Interface Sci. 65, 331 (1978); and Labib, M. E., and Robertson, A. A., J. Colloid Interface Sci. 77, 151 (1980).

15. Versmold, H., Wittig, U., and Härtl, W., J. Phys. Chem. 95, 9937 (1991).

16. Palberg, T., Mönch, W., Bitzer, F., Belloni, L., Bellini, T., and Piazza, R., Helv. Phys. Acta 67(2), 225 (1994).

17. Bucci, S., Fagoti, C., Degiorgio, V., and Piazza, R., Langmuir 7, 824 (1991).

18. Palberg, T., Härtl, W., Wittig. U., Versmold, H., Würth, M., and Simnacher, E, J. Phys. Chem. 96, 8180 (1992).

19. Palberg, T., Kottal, J., Loga, T., Hecht, H., Simnacher, E., Falcoz, F., and Leiderer, P., J. Phys $H I$ (France) 4, 457 (1994).

20. Deggelmann, M., Ph.D. thesis, Universität Konstanz, 1992. 